\title{
A Subtle Link in Switched Dynamical Systems: Saddle-Node Bifurcation Meets Border Collision
}

\author{
Yue $\mathrm{Ma}^{*}$, Chi K. Tse ${ }^{\dagger}$, Takuji Kousaka ${ }^{\ddagger}$ and Hiroshi Kawakami* \\ *Dept. of Electrical and Electronic Engineering Department, The University of Tokushima, Tokushima, Japan \\ ${ }^{\dagger}$ Dept. of Electronic and Information Engineering, Hong Kong Polytechnic University, Hong Kong \\ $\ddagger$ Dept. of Electronic and Electrical Engineering, Fukuyama University, Japan \\ Emails: *mayue(kawakami)@ee.tokushima-u.ac.jp; ${ }^{\dagger}$ encktse@ polyu.edu.hk; ${ }^{\dagger}$ kousaka@ fuee.fukuyama-u.ac.jp
}

\begin{abstract}
Switched dynamical systems are known to exhibit border collision, in which a particular operation is terminated and a new operation is assumed as one or more parameters are varied. In this paper, we report a subtle relation between border collision and saddle-node bifurcation in such systems. Our main finding is that the border collision and the saddle-node bifurcation are actually linked together by unstable solutions which have been generated from the same saddle-node bifurcation. Since unstable solutions are not observable directly, such a subtle relation has not been known. In this paper, we describe an effective method to track solutions regardless of their stability, allowing the subtle phenomenon to be uncovered. Two typical DC-DC converters are observed to verify our finding. ${ }^{1}$
\end{abstract}

\section{INTRODUCTION}

Being a commonly observed phenomenon in switched dynamical systems, border collision has attracted much attention in recent years [1]-[3]. Like other typical bifurcation scenarios, border collision manifests itself as a sudden change of qualitative behavior of a system as one or more parameters are varied, and hence can be regarded as a kind of bifurcation phenomenon. However, border collision has always been considered separately from such traditional bifurcations as saddle-node bifurcation and period-doubling bifurcation. This can be attributed to the fundamental difference in the mechanisms underlying border collision and traditional bifurcations. Specifically, traditional bifurcations are caused by a loss of stability of an operating orbit and the assumption of a new stable orbit, whereas border collision is resulted from operational change in which an operating orbit fails to maintain itself due to some inherent structural property of the system [4]. The system thus typically jumps to another stable operating orbit at border collision. Moreover, no loss of stability is required for border collision. Up to now, because of the apparent lack of commonality between border collision and traditional bifurcations, no connection has been known that links the two types of coexisting bifurcations in a given switched dynamical system.

In our previous work [5], the relation between border collision and period-doubling bifurcation has been discussed briefly. In this paper, we will find a strong link that connects

\footnotetext{
${ }^{1}$ This work was supported in part by the Research Grant Council of Hong Kong under a competitive-bid earmarked research grant (No. PolyU 5241/03E).
}

border collision with saddle-node bifurcation. Specifically, by tracking the border collision that occurs in unstable periodic solutions, we will show how border collision is connected to a coexisting saddle-node bifurcation via unstable solutions. Based on this finding, we are able to explain why and when a "jump" or "continuous transition" occurs during border collision.

\section{SAdDlE-Node BIFURCATION AND BORDER COLLISION}

According to traditional bifurcation theory, if a saddlenode bifurcation occurs, one of the characteristic multipliers of the characteristic equation for a fixed point equals to 1. Such a bifurcation can produce or destroy a pair of solutions: one is a node and the other is a 1-dimensional unstable saddle. Meanwhile, a border collision is a bifurcation phenomenon which is often observed in switched dynamical systems. Unlike traditional bifurcations, border collision is independent of characteristic multipliers. Instead, it occurs when the system experiences a structural change which causes a stable operation to cease, as a result of the system state hitting a spatial or temporal "border" [4]. Specifically, in our previous work [5], a method has been proposed to locate the occurrence of border collision. However, the manifestation of the transition at border collision (i.e., the way in which the system jumps from one orbit to another) is still a complicated problem which is not generally solved.

In much of the previous study, only stable solutions are considered in detail, as limited by the way in which codimension1 bifurcation diagrams are obtained through deriving the steady-state solutions of the system. In the following we will introduce a method to compute the fixed points of switched dynamical systems regarding of their stability. Then, by tracking the bifurcation of unstable solutions, we are able to uncover an important role that unstable solutions play in connecting border collision and saddle-node bifurcation.

\section{Periodic Solutions and Stability}

A switched dynamical system can be described briefly as follows. Consider a simple but general case where a switched dynamical system consists of two sub-systems: $S_{1}: \dot{x}=$ $f\left(x, \lambda_{1}\right)$ and $S_{2}: \dot{x}=g\left(x, \lambda_{2}\right)$, where $x \in R^{n}$ and $\lambda$ 
denote the state variables and system parameters, respectively. Solution flows of the sub-systems are represented by $\varphi\left(t, x_{f 0}\right)$ and $\psi\left(t, x_{g 0}\right)$, where $x_{f 0}$ and $x_{g 0}$ are initial points. Switching is modulated by borders for each sub-system; that is, whenever $\varphi$ or $\psi$ hits the border specifically defined for one sub-system, switching occurs. Suppose borders for $S_{1}$ and $S_{2}$ are given by $B_{1}=\left\{x \in R^{n}: \beta_{1}(x, t)=0\right\}$ and $B_{2}=\left\{x \in R^{n}:\right.$ $\left.\beta_{2}(x, t)=0\right\}$, respectively. Then, the simplest solution flow can be formulated as

$$
\begin{aligned}
& x_{1}=\varphi\left(\tau_{1}, x_{0}\right) \\
& x_{2}=\psi\left(\tau_{2}-\tau_{1}, x_{1}\right) \\
& \beta_{1}\left(x_{1}, \tau_{1}\right)=0 \\
& \beta_{2}\left(x_{2}, \tau_{2}\right)=0
\end{aligned}
$$

where $x_{0}$ is the starting point and $x_{2}$ is the ending point of the flow, and $\tau_{1}$ and $\tau_{2}$ are switching times. Thus, if $x_{2}=x_{0}$, it becomes a periodic solution. Moreover, $x_{1}$ is the ending point of the first interval as well as the starting point of the second interval. Clearly, switching points must satisfy the border functions $\beta_{1}=0$ and $\beta_{2}=0$. Finally, as (1) contains $2 n+2$ scalar equations with $2 n+2$ scalar unknowns, i.e., $\left\{x_{0}, x_{1}, \tau_{1}, \tau_{2}\right\}$, we can solve the periodic solution using an appropriate numerical method. Unstable solutions can be also found since our method does not discriminate the stability status. To determine the stability of the computed periodic solution, one needs to find the Jacobian of the map $F: R^{n} \rightarrow$ $R^{n} ; x_{0} \mapsto x_{2}$, for the solution flow given in (1), and preferably in terms of $\partial x_{2} / \partial x_{0}$.

From the first two equations of (1), we get

$$
\begin{aligned}
& \frac{\partial x_{1}}{\partial x_{0}}=\frac{\partial \varphi}{\partial t} \frac{\partial \tau_{1}}{\partial x_{0}}+\frac{\partial \varphi}{\partial x_{0}} \\
& \frac{\partial x_{2}}{\partial x_{0}}=\frac{\partial \psi}{\partial t} \frac{\partial \tau_{2}}{\partial x_{0}}-\frac{\partial \psi}{\partial t} \frac{\partial \tau_{1}}{\partial x_{0}}+\frac{\partial \psi}{\partial x_{1}} \frac{\partial x_{1}}{\partial x_{0}}
\end{aligned}
$$

where $\varphi$ and $\psi$ denote $\varphi\left(\tau_{1}, x_{0}\right)$ and $\psi\left(\tau_{2}-\tau_{1}, x_{1}\right)$, respectively. Moreover, from the border functions (i.e., last two equations in (1)), we have

$$
\begin{aligned}
& \frac{\partial \beta_{1}}{\partial x_{0}}=\frac{\partial \beta_{1}}{\partial x} \frac{\partial x_{1}}{\partial x_{0}}+\frac{\partial \beta_{1}}{\partial t} \frac{\partial \tau_{1}}{\partial x_{0}}=0 \\
& \frac{\partial \beta_{2}}{\partial x_{0}}=\frac{\partial \beta_{2}}{\partial x} \frac{\partial x_{2}}{\partial x_{0}}+\frac{\partial \beta_{2}}{\partial t} \frac{\partial \tau_{2}}{\partial x_{0}}=0
\end{aligned}
$$

Hence, $\partial \tau_{2} / \partial x_{0}, \partial \tau_{1} / \partial x_{0}$ and $\partial x_{1} / \partial x_{0}$ can be obtained easily. Direct substitution yields

$$
\frac{\partial x_{2}}{\partial x_{0}}=\frac{\left(\frac{\partial \psi}{\partial x_{1}} \frac{\partial \beta_{1}}{\partial t}+\frac{\partial \psi}{\partial t} \frac{\partial \beta_{1}}{\partial x}\right) \frac{\partial \varphi}{\partial x_{0}} \frac{\partial \beta_{2}}{\partial t}}{\left(\frac{\partial \beta_{2}}{\partial t}+\frac{\partial \psi}{\partial t} \frac{\partial \beta_{2}}{\partial x}\right)\left(\frac{\partial \beta_{1}}{\partial t}+\frac{\partial \varphi}{\partial t} \frac{\partial \beta_{1}}{\partial x}\right)}
$$

All terms in the above equation can be calculated by an appropriate numerical method. Thus, by finding the roots of the characteristic equation, we can determine the stability of any periodic solution.

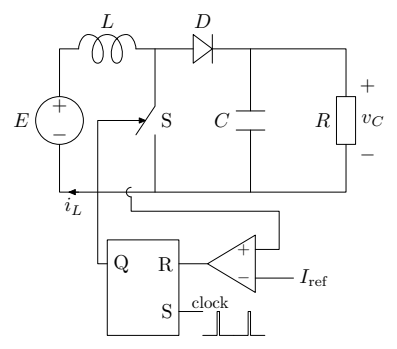

(a)

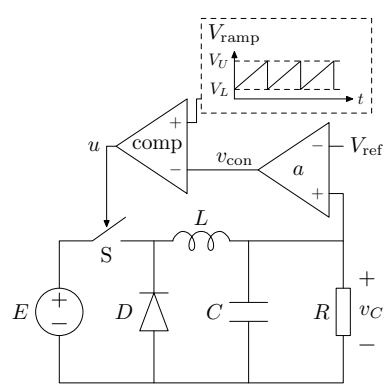

(b)
Fig. 1. (a) Current-mode controlled boost converter; (b) Voltage-mode controlled buck converter.

\section{Illustration OF MAIN Findings}

In much of the previous study, border collision has been shown to exhibit a "jump" in the bifurcation diagram, as a stable operating orbit suddenly gives way to another stable orbit in a discontinuous fashion. Border collision "terminates" a specific solution and the system "jumps" to another attractor. The resulting solution is entirely new and seems to have little relation with the original solution assumed before the onset of border collision. In the following, we will illustrate that the new solution is often generated by a saddle-node bifurcation. In fact, saddle-node bifurcation gives birth to a node and a saddle. While the node can be directly observed, the saddle (or the unstable solution) is invisible and much less considered.

The problem is best illustrated with examples. We will consider the current-mode controlled boost dc-dc converter and the voltage-mode controlled buck dc-dc converter. They provide very accessible examples on explaining the subtle connection between the coexisting border collision and saddlenode bifurcation.

\section{Example 1: Current-Mode Controlled Boost Converter}

In the current-mode controlled boost converter circuit shown in Fig. 1(a) [7], the switch is turned on periodically and turned off whenever the current of the inductor reaches a reference value. Thus, the two sub-systems are given by

$$
\begin{array}{ll}
S_{1}: & \dot{x}=\left[\begin{array}{cc}
-1 / R C & 0 \\
0 & 0
\end{array}\right] x+\left[\begin{array}{c}
0 \\
1 / L
\end{array}\right] E \\
S_{2} & : \quad \dot{x}=\left[\begin{array}{cc}
-1 / R C & 1 / C \\
-1 / L & 0
\end{array}\right] x+\left[\begin{array}{c}
0 \\
1 / L
\end{array}\right] E .
\end{array}
$$

When it works in continuous conduction mode, the two borders can be described by

$$
\begin{aligned}
& B_{1}=\left\{(x, t) \in R^{2} \times R: \beta_{1}=i_{L}-I_{\text {ref }}=0\right\} \\
& B_{2}=\left\{(x, t) \in R^{2} \times R: \beta_{2}=t-k T=0\right\}
\end{aligned}
$$

where $x=\left[\begin{array}{ll}v_{C} & i_{L}\end{array}\right]^{T}, k=1,2, \cdots$. A typical one-parameter bifurcation diagram is shown in Fig. 2, with the reference current $I_{\text {ref }}$ being the bifurcation parameter and the sampled $i_{L}$ being the variable. Here, as revealed from the blow-up views, as $I_{\text {ref }}$ increases, border collision takes place to terminate the period-2 solution, and the system jumps to a period-4 solution. Moreover, if we move backward (decreasing $I_{\text {ref }}$ ), we see that 


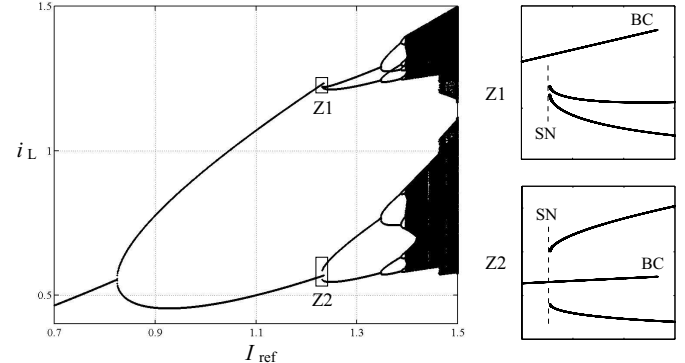

Fig. 2. One-parameter bifurcation diagram of current-mode controlled boost converter, with two blow-up views of regions z1 and z2. Parameters are $R=$ $40 \Omega, L=1.5 \mathrm{mH}, T=100 \mu \mathrm{s}, \tau_{C}=R C / T=2$ and $E=10 \mathrm{~V}$.

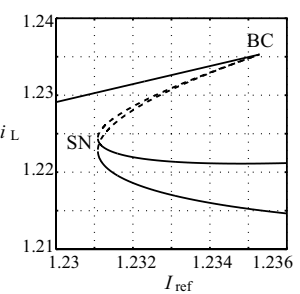

(Z1)

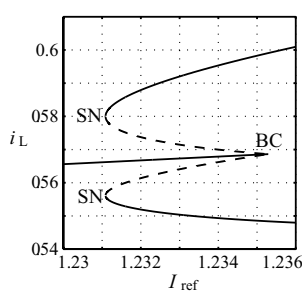

(Z2)
Fig. 3. Blow-up views of the one-parameter bifurcation diagram showing bifurcations of unstable solutions. Parameters are same as those in Fig. 2.

the period-4 solution ends up at a saddle-node bifurcation, and the solution jumps back to the original period-2 solution.

Although only a stable period-4 solution is observed, an unstable period-4 solution exists. This unstable period-4 solution, being unobservable, has been rarely noticed, let alone discussed. Using the method introduced previously, however, we can track the position of the unstable solutions easily. The results are shown in Fig. 3, where unstable period-4 solutions are traced and shown as dashed curves.

The key message of Fig. 3 is that unstable solutions are also terminated by the same border collision. In other words, the stable period- 2 solution and the unstable period- 4 solution are terminated by the same border collision, but in opposite parametric directions. Thus, we see that the border collision is actually being linked to the saddle-node bifurcation by the unstable period-4 solution.

\section{Example 2: Voltage-Mode Controlled Buck Converter}

We now consider a voltage-mode controlled buck converter, as shown in Fig. 1(b). A feedback signal, $V_{\text {con }}$, is compared with a ramp voltage signal, $V_{\text {ramp, }}$, to control the switch. Therefore, we can write the two sub-systems as

$$
\begin{array}{ll}
S_{1}: & : \dot{x}=\left[\begin{array}{cc}
-1 / R C & 1 / C \\
-1 / L & 0
\end{array}\right] x \\
S_{2} & : \quad \dot{x}=\left[\begin{array}{cc}
-1 / R C & 1 / C \\
-1 / L & 0
\end{array}\right] x+\left[\begin{array}{c}
0 \\
1 / L
\end{array}\right] E
\end{array}
$$

where $x=\left[\begin{array}{ll}v_{C} & i_{L}\end{array}\right]^{T}$. The border of this system is defined as

$$
\begin{aligned}
\beta_{1}(x, t) & =\beta_{2}(x, t) \\
& =a\left(v_{C}-V_{\text {ref }}\right)-\frac{\left(V_{U}-V_{L}\right) t}{T}-V_{L}=0
\end{aligned}
$$

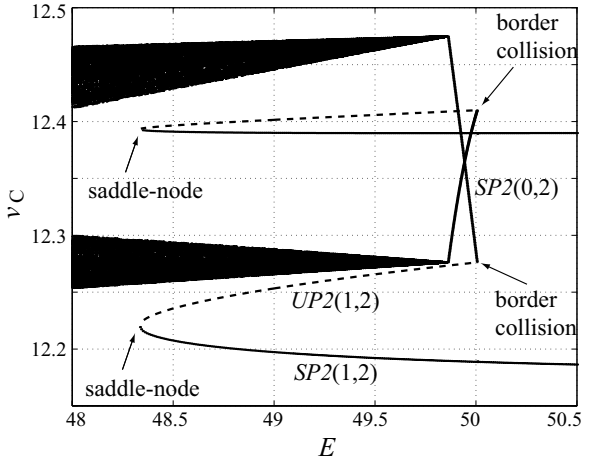

Fig. 4. One-parameter bifurcation diagram from voltage-mode controlled buck converter. Parameters are $T=400 \mu \mathrm{s}, L=20 \mathrm{mH}, a=8.4, V_{\text {ref }}=$ $11.3 \mathrm{~V}, V_{L}=3.8 \mathrm{~V}, V_{U}=8.2 \mathrm{~V}$ and $C=47 \mu \mathrm{F}$.

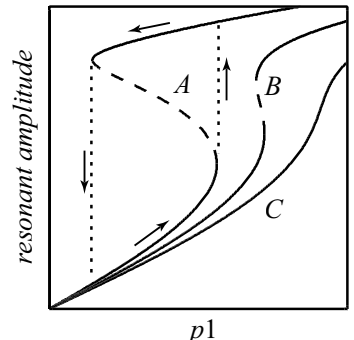

(a)

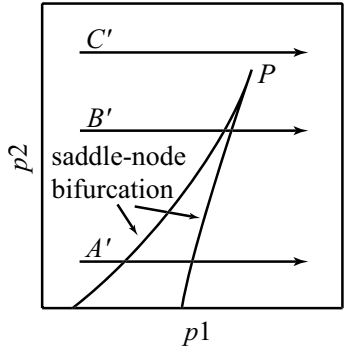

(b)
Fig. 5. Illustration of jump and hysteresis phenomena. (a) One-parameter bifurcation diagram; (b) two-parameter bifurcation diagram. Varying parameter $p 1$ along lines $A, B$ and $C$ in (b), we get three curves of resonant amplitude characteristics in (a).

for $t \in[0, T]$, which is a common border for both two subsystems.

Our previous studies [5] have shown that a period-2 solution can jump to another stable period-2 solution via a border collision, as shown in Fig. 4. Here, we track the unstable period-2 solution generated from saddle-node bifurcation, and observe a similar phenomenon. As denoted by dashed curves in Fig. 4, the unstable solution conjoins with the stable solution at the border collision point.

\section{Details of the Bifurcation Connection And INTERACTION}

In the foregoing, we have described how stable solutions connected to different bifurcations are tied together by unstable solutions. This is similar to the phenomenon of hysteresis, which is often found in systems with resonating states associated with saddle-node bifurcation. At this point, it is instructive to recall the familiar behavior of the Duffing's equation shown simplistically in Fig. 5. Here, curves $A, B$ and $C$ indicate the resonant amplitude characteristics corresponding to movements of parameters along the horizontal lines $A^{\prime}$, $B^{\prime}$ and $C^{\prime}$. In the case of parameter movement along $A^{\prime}$, solution moves along the solid curves in the arrow direction in Fig. 5 (a). When it comes to the point with vertical tangency, a slight change of $p 1$ will cause a discontinuous jump of the amplitude to the upper or lower portion of the curve. Between 


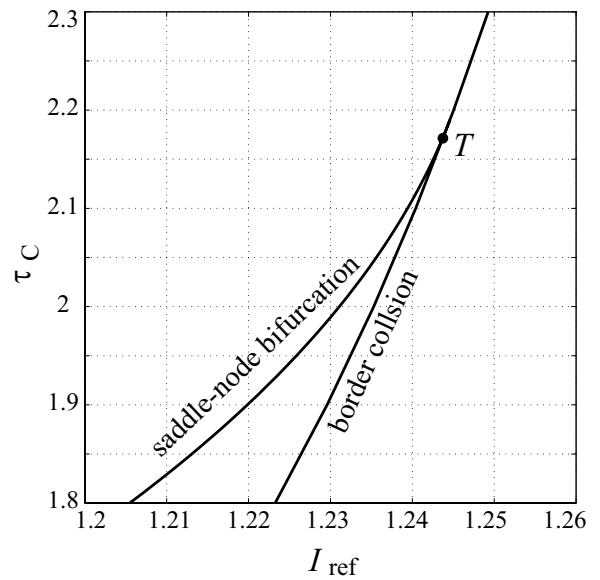

Fig. 6. Bifurcation diagram of boost converter with same set of parameters as Fig. 2.

the two bifurcation curves in Fig. 5 (b), coexistence of the two solutions is possible. Moreover, as we increase $p 2$, the two bifurcation curves in Fig. 5 (b) will move closer to each other. For example, along line $B^{\prime}$, the coexistence region is much narrower than that along line $A$. Finally, for the case of movement along line $C^{\prime}$, i.e., beyond the cusp point $P$ of the bifurcation curves, no bifurcation occurs for the solution and coexistence is no longer observed.

Returning to our switched systems, from Figs. 3 and 4, we see that saddle-node bifurcation and border collision are articulated in a similar manner as the aforementioned jump and hysteresis phenomenon.

We now take a detailed look at the bifurcation behavior of the current-mode controlled boost converter around the region where border collision and saddle-node bifurcation occur. Fig. 6 shows the bifurcation diagram in the $\left(I_{\text {ref }}, \tau_{C}\right)$ plane. Here, we see that the saddle-node bifurcation curve merges tangentially with the border collision curve at point $T$. Stable period-2 solutions exist on the left-hand side of the border collision curve and stable period-4 solutions exist on the right-hand side of the saddle-node bifurcation curve. Thus, between the two bifurcation curves, coexistence and hysteresis can be observed, as shown in Fig. 3, where $\tau_{C}=2$. Similar to the situation illustrated by the Duffing's system (Fig. 5), increasing $\tau_{C}$ above the tangent point $T$ will make saddlenode bifurcation vanish. However, the border collision still remains. If we move parameter $I_{\text {ref }}$ along the line $\tau_{C}=2.3$, we get a one-parameter bifurcation diagram, as shown in Fig. 7. Comparing with Fig. 3, we see that unstable solutions disappear due to the tangential merger of border collision and saddle-node bifurcation. Finally, the scenes shown in Fig. 7 look deceptively like a period-doubling bifurcation. Here, it is clear that they are the results of the interaction between saddle-node bifurcation and border collision, corresponding to the choice of parameters above the tangent point $T$.

Similar phenomena can also be observed in the buck converter. As $R$ is decreased, the saddle-node bifurcation will

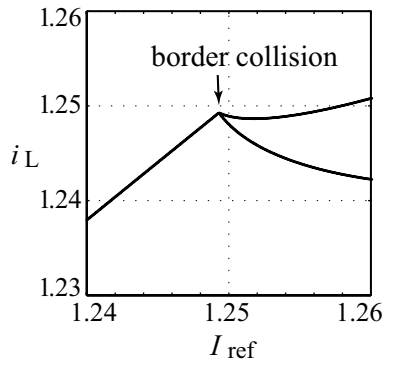

(a)

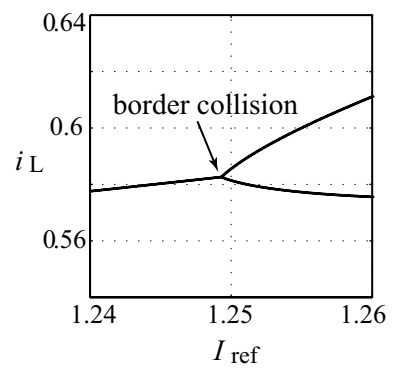

(b)
Fig. 7. One-parameter bifurcation diagrams showing no hysteresis jump, with $\tau_{C}=2.3$. This corresponds to the case above the tangent point $T$ in Fig. 2.

merge tangentially with the border collision (see Fig. 4), and the two period- 2 solutions will be connected together. This clearly explains why border collision may manifest itself as a jump (hysteresis) or as a continuous turning point, depending upon the parameter values being below or above the tangent (merging) point.

\section{CONCLUSION}

Being fundamentally different in the underlying bifurcation mechanisms, border collision and saddle-node bifurcation have rarely been considered in a unified way. In this paper we have discussed the relation between border collision and saddlenode bifurcation, and identified the way in which the two bifurcations are connected. The key finding is that border collision is connected to saddle-node bifurcation via unstable solutions that have been generated by the same saddlenode bifurcation. This explains the jump phenomenon that occurs at border collision. Moreover, as parameters vary, the border collision and the saddle-node bifurcation points merge tangentially. Beyond this merger point, border collision no longer manifests as jumps, but rather as continuous transitions which typically resemble turning points with possible periodmultiplying in one-parameter bifurcation diagrams.

\section{REFERENCES}

[1] H. Nusse, E.Ott, and J. Yorke, "Border-collision bifurcations: an explanation for observed bifurcation phenomena," Physical Review E, vol. 49, pp. 1073-1076, 1994.

[2] S. Banerjee, P. Ranjan, and C. Grebogi, "Bifurcation in two-dimensional piecewise smooth maps - theory and applications in switching circuits," IEEE Transactions on Circuits and Systems Part I, vol. 47, no. 5, pp. 633-643, May 2000.

[3] M. di Bernardo, C. J. Budd, and A. R. Champneys, "Grazing and bordercollision in piecewise-smooth systems: a unified analytical framework," Physical Review Letters, vol. 86, no. 12, pp. 2553-2556, March 2001.

[4] C. K. Tse, Complex Behavior of Switching Power Converters. Boca Raton: CRC Press, 2003.

[5] Y. Ma, H. Kawakami, and C. K. Tse, "Analysis of bifurcation in switched dynamical systmes with periodically moving borders," IEEE Transactions on Circuit ans Systems: Part I, vol. 51, no. 6, pp. 1184-1193, June 2004.

[6] C. K. Tse and M. di Bernardo, "Complex behavior of switching power converters," Proc. IEEE, vol. 90, no. 5, pp. 768-781, 2002.

[7] W. C. Y. Chan and C. K. Tse, "Study of bifurcations in currentprogrammed $\mathrm{dc} / \mathrm{dc}$ boost converter: From quasi-periodicity to perioddoubling," IEEE Transactions on Circuits and Systems Part I, vol. 44, no. 12, pp. 1129-1142, 1997. 